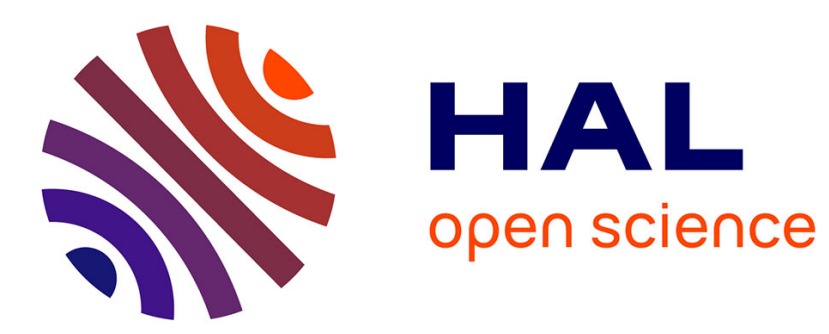

\title{
Analysis of self-reconfigurable modular systems, a design proposal for multi-modes locomotion
}

Nicolas Brener, Faiz Ben Amar, Philippe Bidaud

\section{To cite this version:}

Nicolas Brener, Faiz Ben Amar, Philippe Bidaud. Analysis of self-reconfigurable modular systems, a design proposal for multi-modes locomotion. IEEE International Conference on Robotics and Automatio, 2004, La Nouvelle Orléans, United States. pp.996-1001. hal-03178087

\section{HAL Id: hal-03178087 \\ https://hal.science/hal-03178087}

Submitted on 23 Mar 2021

HAL is a multi-disciplinary open access archive for the deposit and dissemination of scientific research documents, whether they are published or not. The documents may come from teaching and research institutions in France or abroad, or from public or private research centers.
L'archive ouverte pluridisciplinaire HAL, est destinée au dépôt et à la diffusion de documents scientifiques de niveau recherche, publiés ou non, émanant des établissements d'enseignement et de recherche français ou étrangers, des laboratoires publics ou privés. 


\title{
Analysis of Self-Reconfigurable Modular Systems A Design Proposal for Multi-Modes Locomotion*
}

\author{
Nicolas Brener, Faïz BenAmar, Philippe Bidaud \\ Laboratoire de Robotique de Paris \\ Université Pierre et Marie Curie Paris VI \\ 18, route du Panorama, 92265 Fontenay aux Roses - France \\ \{brener,amar,bidaud\}@robot.jussieu.fr
}

\begin{abstract}
This paper presents some general considerations on self-reconfigurable robots design, and proposes an original design of mechatronic modules. Geometrical and kinematical features of these modules, offer the ability to be used as well as wheels to produce rolling motion, and as joints for building kinematic chains as legs, arms or snakes.
\end{abstract}

Keywords : modular, self-reconfigurable.

\section{INTRODUCTION}

In the last years, many efforts have been made in research in modular robotics and especially in self-reconfigurable systems (SRS), i.e. systems which are able to change dynamically their topology. Such systems are made of sets of mechatronic building blocs which have the capacity to connect and disconnect together. By manipulating themselves their own building blocs, those systems can change their topology. The advantages of modular systems are several:

- $\quad$ Easy and fast to deploy: the robot does not need to be designed and constructed, the modules are already available and can be fast and easily manually assembled. The required topology for a task is automatically generated by a software and the control algorithm is downloaded to the robot.

- $\quad$ Easy and fast maintain: a deficient module can be easy and fast replaced.

- High versatility and few stuff: a set of modules can be assembled in various topologies optimised for various tasks.

- Adaptability: it is possible to perform tasks which were not originally foreseen.

- Low cost: the genericity of the modules authorizes mass-production

Furthermore, a self-reconfigurable system has even more advantages:

- Autonomy: the robot can reconfigure and repair itself

The applications of SRS are numerous: i.e. industrial manipulations, locomotion on rough and difficult terrain, pipe inspection, space station construction, etc.

Because of their versatility and robustness selfreconfigurable systems are very interesting for space applications. A set of robotic modules could do a very wide range of tasks, which would else need numerous big and heavy non-modular robots, including tasks which are not originally foreseen. Moreover a low gravity context reduces tremendously the mechanical constraints and permits much more efficient SRS. The system can move more economically, carry heavier loads and form bigger structures. All candidate sites in space, like orbital stations, asteroids, moons, and non-gaseous planets, have less gravity than on Earth.

The mechatronic design of self-reconfigurable robotic modules is quite complex because it involves a high degree of technology for energy storage or production, efficient and low energy consumption of the connexion mechanism, distributed control system, etc.

The optimisation of module characteristics (like connectivity, mobility, geometry) which gives the best versatility is a non-trivial problem because they are only components of a modular systems which should be able to form an undefined number of different topologies, depending of the task and the context, like the local shape of the terrain. Therefore the modules design must be optimised for some main and general functions which must be defined first.

In section II and III we introduce some characteristics of SRS and application classes. Section IV analyses how to increase the versatility and efficiency of these systems. An original design is presented in section $\mathrm{V}$ and discussed in section VI.

\section{TERMINOLOGY AND DEFINITIONS}

This section reviews some properties of SRS. These are recapitulated in Table 1.

\section{A. Matricial-type SRS vs chain-type SRS}

In matricial type SRS, the modules have discrete positions/orientations and are localized in a virtual regular grid, like a tree-dimensional chessboard, bee structure, or other. A reconfiguration of these kind of system, consist on discrete motions, typically $\pm 90^{\circ}$ rotation, proceeded step by step while the assembly remains globally connected. At each step some blocs are moved at an adjacent place on the grid. Depending on the geometry and configuration of the SRS, a module can move itself from a case to an adjacent one, or need the help of adjacent modules. Examples of such SRS are Molecule[1], MTRAN[4], I-Cube[5], Telecube [6], ], Micro-Unit [7].

In chain type SRS, the position and motion of the modules are no more discrete. Therefore reconfiguration is more complex and it can need up to $6 \mathrm{DOF}$, provided by a chain manipulator, to move a module at a desired place. Conro and 
PolyBot are typical chain type SRS. Some SRS like MTRAN[4] are both types.

\section{B. Homogeneity, heterogeneity}

If a SRS is formed only by one type of module, it is homogeneous, else it is heterogeneous. In heterogeneous systems the ratio of different module types is variable. In some heterogeneous systems, the ratio can not be changed, because of kinematical and geometrical properties. An example is the heterogeneous version of the Molecule [1] robot. We can call these pseudo-homogeneous.

\section{Connectivity}

Connectivity defines the number and type of connection devices on the modules. Different connection systems for SRS are proposed in the literature. Some of them have genderless connectors said also universal connectors, other male and female connectors. For homogeneous systems, as described in [2]:

- Open or closed chains need at least two universal connectors or one male and one female connector.

- Graphs containing at most one cycle with an arbitrary number of forks, like trees, need at least one male and two female connectors.

- Arbitrary graph topologies need at least three universal connectors, or two male and two female per module.

At long-term, continuous connection systems without connection plates can be envisaged as described in [3].

\section{Isotropy and anisotropy}

If all the directing vectors of the mobility axes of a module are in the same plane (except in singular configurations), the module is said anisotropic, otherwise it is said isotropic.

Similarly, if all the directing vectors of the mobilities of an assembly are in the same plane (except in singular configurations), the assembly is said anisotropic, otherwise it is said isotropic. An anisotropic assembly can not reconfigure itself and lonely in an isotropic one, the reciprocal is also true. Therefore anisotropy reduces severely the reconfiguration capabilities of an assembly. Notice that an istotropic assembly can be formed with anistropic modules (it is sufficient that two anisotropic modules have their directing axis in different planes). A complete review of these properties can be found in [4] about the M-TRAN robot.

\section{E. Topological and kinematical reconfigurations.}

In a topological reconfiguration at least one module connects or disconnects. If an assembly changes its shape and behaviour without any connection or disconnection, it does a kinematical reconfiguration.

\section{ClASSIFICATION}

We have identified four main applications classes for SRS.

\section{A. Shape-transforming}

Shape-tranforming is the ability to form static structures which can change their shape by reconfiguration.
It permits to make self-transformable home furniture, adaptive tools, automatic growing of buildings. More realistic application at short-term is for space station by using modular SRS which allow automatic self assembling and topology changing when it is needed.

\section{B. Manipulation}

SRS can perform manipulation like classical robots. We can distinguish:

- Linear manipulator, using a linear chain of modules. Chain-type SRS are well suited to perform such manipulators.

- $\quad$ Parallel manipulator, using a closed chain of modules.

- Truss manipulator, which is formed of several layers of modules. In this way, it is possible to adapt the diameter of the manipulator: it can be longer, stronger and faster than a simple chain manipulator. Nevertheless such deformable trusses are difficult to construct due to kinematics and collision constraints of the structure. These systems are also difficult to control. The modular robot Tetrobot [8] can form such manipulators, but it has to be reconfigured manually. If a gripper is needed, it must be provided by a specialized module.

- Distributed manipulation, where several manipulators cooperate to move an object, or one assembly interacts with an object at several points.

\section{Exploration}

Exploration needs locomotion ability. In some SRS, modules can move individually (see $[9,16]$ ), but it is, in general, not the case for the others. To provide a displacement, a SRS can use the same topologies as for manipulation: forming legs or arms (for climbing) that can be seen as distributed manipulators, serial chains can also move by using reptation or caterpillar gaits [10]. Closed chains can perform wheel motion, as well as deformable trusses like rolling ball with the Tetrobot [8]. Another typical locomotion technique is to use reconfiguration: the modules at the rear of an assembly continuously migrate at the front of it, as shown in $[1,4,5]$ with the Molecule, M-TRAN and I-Cubes systems. This locomotion, based on some circulation of discrete elements, can be approached to tracked locomotion.

It is also possible to use specialized modules with wheels or hybrid locomotion with wheeled legs.

\section{Transport}

Transport needs locomotion like for exploration, but also carrying ability. We can distinguish the case where a mobile assembly carries an object which is static relatively to it, and the case were the assembly is static relatively to the ground, but moves an object. This second case is similar with distributed manipulation. Hybrid techniques are also possible, like "moonwalk" with the Polypod SRS detailed in [2]. 
TABLE I. PROPERTIES OF SOME SRS

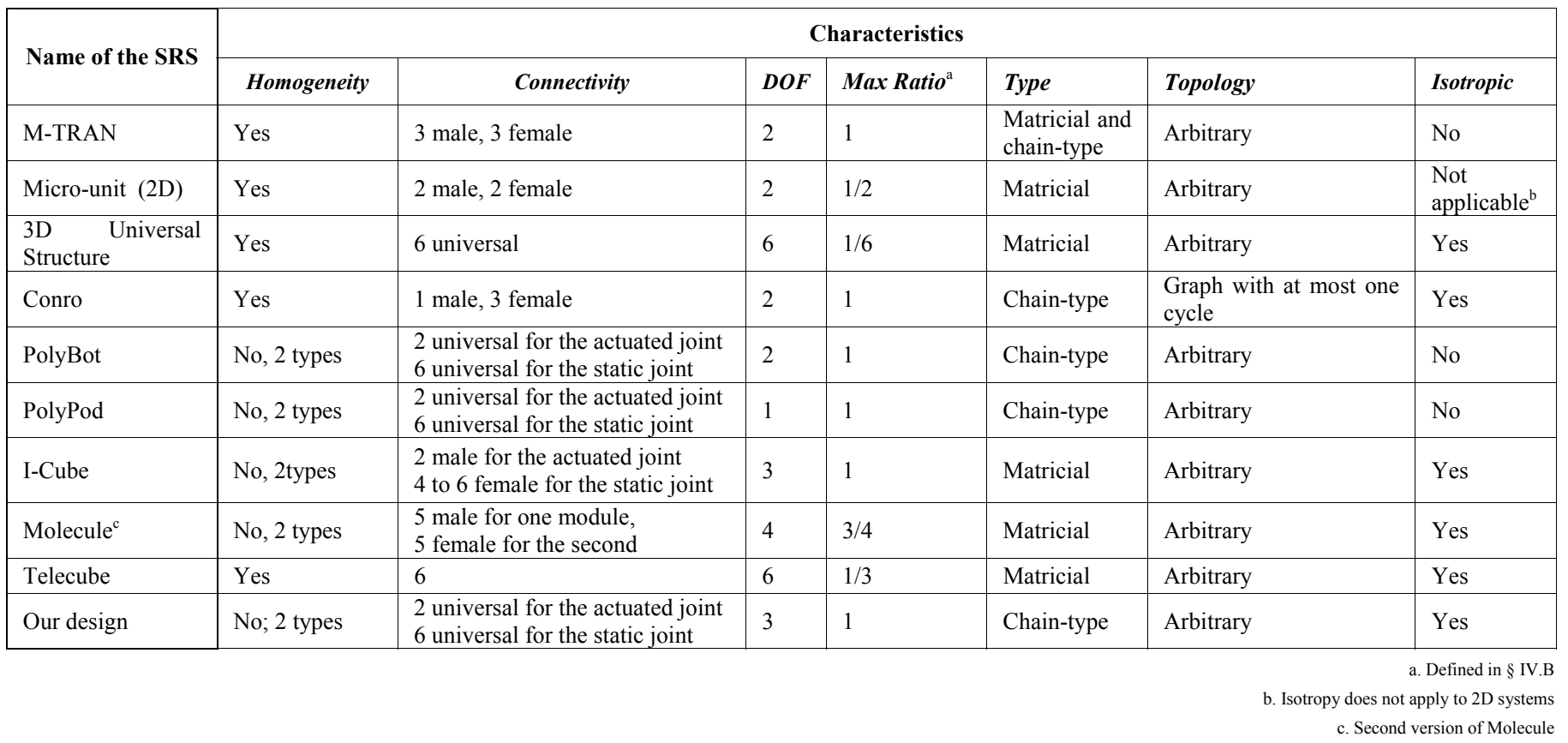

\section{DESIGN}

\section{A. Homogeneity and versatility}

A self-reconfigurable system needs actuators, low-level control hardware, internal sensors, and connexion mechanisms. Autonomous mobile SRS need also energy source, high level control software, wireless telecommunications systems, localisation sensors, vision sensors, etc... To increase the versatility, numerous specialized devices, like grippers, work tools, and various sensors are necessary. It is not possible to put all the devices in each module, because they would become too big. A better approach is to distribute the specialized devices among the set of modules. This means that a versatile system should not be strictly homogeneous. However functional heterogeneity of the modules does not imply geometrical heterogeneity: the modules can be specialized by putting the various devices in a generic subpart of each module, without changing their kinematical and geometrical properties. On this way the motion and reconfiguration capabilities of the assembly are preserved, and it can put the proper components at the proper places by reconfiguration. Since some of the devices need extern access, it is necessary to put the generic subpart somewhere at the border of each module, this makes also easier to replace the devices by others. Small devices can be packed together in one component which is plugged into the dedicated subpart of a module.

However geometrical and kinematical heterogeneity of the modules can also be useful since it allows the optimisation of the kinematics of an assembly by combining, for example, kinematic and static joints in an efficient way for a particular task. Moreover, it is not very probable that in a large and very versatile SRS, it is possible to pack all the, various sized and shaped, specialized devices in one geometry type of module. On the contrary, having several modules geometry diminish the genericity, the robustness and reconfigurability of the system.
We conclude that a good compromise is to have only some modules with different geometry or kinematics and many devices distributed redundantly in the entire set of modules, their redundancy depending on their use probability and importance. To maximize the robustness (fault-tolerance) every device should be present at least twice.

\section{B. Actuation}

One major problem with the SRS is their mechanical efficiency. Purely shape-transforming matricial systems do not need a high torque or force to reconfigure. Other versatile systems, especially chain-type ones, where the system must interact with the environment, the motion of the modules needs more torque or force because they have to counteract extern constraints. Contrary to classical robots, SRS must integrate connecting mechanisms, which have a non-negligible weight. They also have predetermined joint kinematics, thus, for a particular motion, a self-reconfigurable chain manipulator can not be generally as good optimised as a dedicated classical one.

To increase the mechanical efficiency of the system it is important to reduce its weight by reducing the number of actuators. Let us consider a module with 6 connexion plates and one actuated mobility per plate. The mobility of a closed chain formed by $n$ modules is at most equal to $2 n$, while its number of actuators is equal to $6 n$, thus $4 n$ actuators are not used and are useless in this case. One could argue that it is possible to have several DOF with only one motor by using magnetic clutches, or differentials with locking systems, but even these mechanisms are strongly space and weight consuming. To reduce the number of actuators, without decreasing the number of mobilities of the closed chain, there must exist at least two connexion plates between which the mobility is equal to the total number of DOF of the module. That way it is possible to form an articulated closed chain without any unused actuators. Even in this case, if two actuated 
plates, connected together, have a coaxial rotation axis, both mobilities become one. Let us consider $N$ the total number of DOF of a closed chain, and $n$ the number of actuators used in a closed chain, minus the coaxial rotary redundancies between connected plates. The column "Max Ratio" of Table 1 shows the maximal ratio $n / N$ for various SRS. As we can see, all chain-type SRS like Conro[14], Polypod[2], and Polybot[11] have a ratio equal to 1 , which is optimal. Some matricial SRS, like I-Cube[5] and M-TRAN[4] (which is both matricial and chain- type) have a ratio equal to 1 too. Nevertheless, most of today's matricial SRS $([1,5,6,7])$ have a ratio less than 1 .

Another way to increase the efficiency is to have the possibility to lock the joints by using irreversible motion transmission or brakes. That way, it is possible to maintain an actuator in a constant position without energy consumption. Only the transition between locked and released states should need some energy (see [10]). Moreover locking mechanisms can support much more constraints than active ones. These mechanisms can also be exploited to change dynamically (without topological reconfiguration) the Jacobian of a kinematical chain and optimise it during a motion depending of the extern constraints. With closed chains, locking mechanisms can lead to large mechanical advantages near singular configurations as explained in [10].

\section{A Design Proposal}

(a)

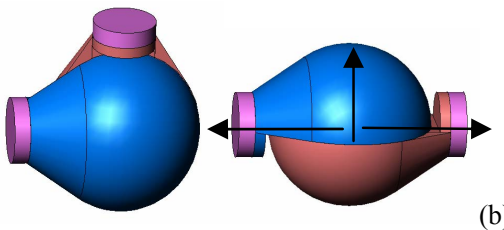

(b) (c)

Figure 4. The ball and the cube modules. (a) a ball in its full range configuration. (b) a ball in wheel configuration, the arrows show the mobility axis, (c) a cube

We will present in this section a new design modules for a SRS where the kinematics and geometry of the modules allows intrinsic wheel motions, without the need of specialized wheeled units. It can produce low consumption locomotion and easily move huge assemblies or carry heavy loads on flat terrain. The proposed design has only been simulated so far, using the ODE (Open Dynamics Engine) library for C programming language.

\section{A. Kinematics and Geometry}

The system consists of two modules:

- A passive cube with 6 universal connecting plates, that can be used as fork joint

- An actuated ball joint with three concurrent axis between two universal connecting plates. Fig. 1 shows a cube and a ball in its two singular particular configurations. Both external joint have no stops and can rotate continuously.

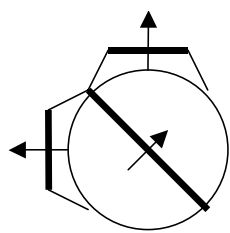

(a)

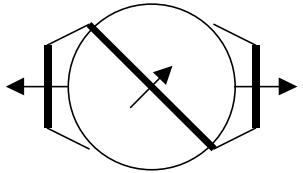

(b)
Figure 2. Alternate design of the ball in (a) its full range configuration and in (b) wheel configuration. The arrows show the mobility axis

An alternate design (Fig 2) consists in using a 45 degree joint in the middle. Notice that, by coordinating its three rotations, this joint can perform plane bending (the joint bends in one plane) and orienting (the joint orients the bending plane), as well as the first design. Both designs have the same workspace which is equal to a half sphere, and can be used as wheels when they are attached on the opposite sides of a cube. Fig. 3 shows two rovers which use the balls as wheels.
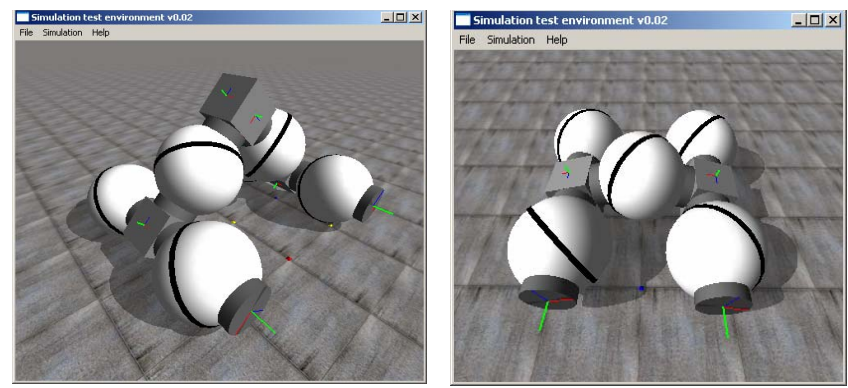

Figure 1. Two rover topologies

\section{B. Simple Topology Examples}

Connecting serially several cubes and balls provides a serial manipulator, with one end fixed to the ground or to a large static assembly. A linear manipulator (Fig. 4a) where the balls are attached on opposite faces of the cubes does not exploit all the mobilities because the revolute joints fixed on opposite

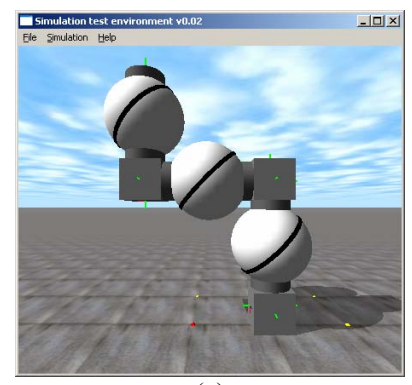

(a)

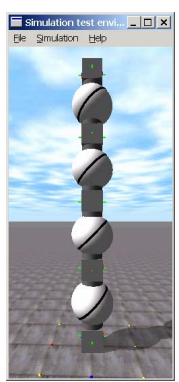

(b)

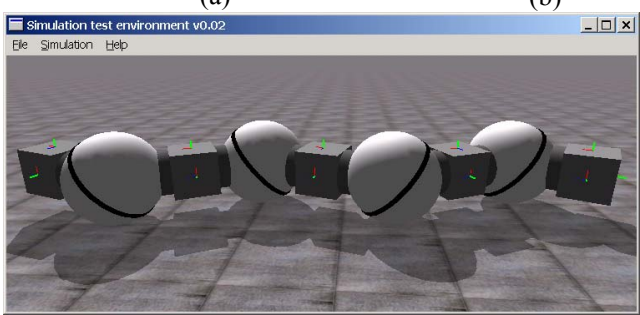

(c)
Figure 3. An orthogonal manipulator (a), a linear manipulator (b), and a snake (c) 


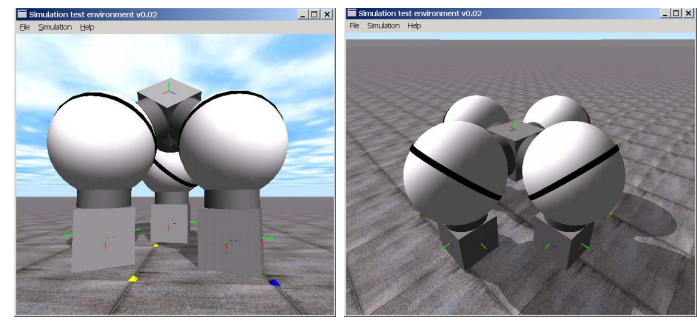

Figure 5. Left a Tripod and right a Quadruped

faces of the cubes are redundant. On contrary, an orthogonal arm (Fig. 4b), where the ball are fixed on adjacent faces of the cubes, uses all the mobilities. Snakes can be formed with the same topologies (Fig. 4c) for moving in small tunnels, by using wave motion. Tripods and quadrupeds can be easily constructed as shown on Fig. 5, as well as hexapods (Fig. 7d). Fig 6a shows a Carpet topology, where the cube and the balls are alternately connected in a plane. When contracted in the Y direction (Fig 6b), only the balls oriented in the $\mathrm{X}$ direction touch the ground, therefore the carpet can move in the $\mathrm{Y}$ direction by using the roll motion of the balls oriented in the $\mathrm{X}$ direction. While moving, the carpet can carry a payload laying on its top. The contrary is also possible: the carpet is static on the ground and moves a payload on the top of it.

\section{Topological and Kinematical Reconfiguration}

\section{1) Kinematical Reconfiguration}

Fig. 7 represents two examples of kinematical reconfiguration. The first represents a reconfiguration from quadruped to a rover by changing the axis of its feet. The second illustrates the reconfiguration from an hexapod to a rover.

\section{2) Topological Reconfiguration}

This system is chain-type, it uses kinematical chains to manipulate modules and move them at their new place. Fig. 8 shows the stages of a reconfiguration from Rover to Snake which needs two connections and two disconnections.

\section{DISCUSSION}

The proposed design has some similarities to the Polybot robot, both are heterogeneous chain-type systems, having two type of modules, one actuated with two connecting plates, and one passive cubic module with several connectors which is used as fork joint. The properties of our design are summarized in Table 1. The main difference is that the revolute joint is replaced by a ball joint. Notice that the same revolute motion as the Polybot can be done with our design. But this design has more reconfiguration ability because, (1) it is isotropic and (2)
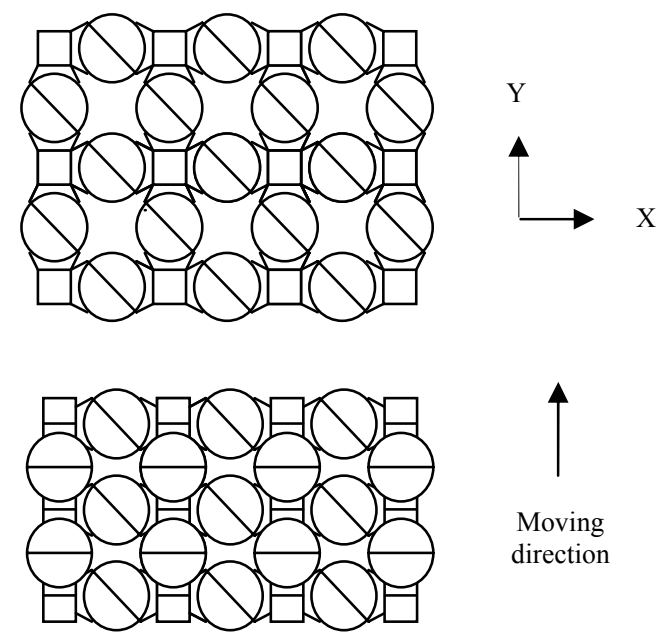

(a)

Figure 6. (a) a carpet, (b) a contracted rolling carpet

(b)

the higher mobility of the actuated joints allows to use less of them, and in return to use more static fork joints, which are important for reconfiguration. One other advantage is the rolling motion of the ball joint. For connecting, we plan to use the same universal connection mechanism as for Polybot: plates with pins and holes, and SMA actuated latches [13].

More problematic is the internal energy and data exchange between a ball and its connecting plates, since stop free rotation of them is necessary for wheel motion. The energy can be stored in an internal battery in each module, however, connected module must be able to exchange energy through the actuated plate because (1) it permits a module to get energy by a neighbor, when its battery is weak, (2) an assembly must be able to reload its batteries by connecting only one module on an energy source. This can be done by using brush mechanisms. Data can be transferred by firewire Ethernet directly from a module to another. However, stop free rotation has also an advantage: if we considerer two chains with the same kinematics, the first with only stop free revolute joints and the second with only 360 degree joints, both have the same workspace but the first has a greater number of path choices between two configurations.

Two questions are still open: the connectivity of the cubes and the actuation mechanism of the balls. Having 6 universal connectors on the cube is probably not necessary. To increase the versatility of the system we plan to reduce the connectivity of the cubes and keep at least one face of the cubes to put specialized components. Some of the connectors could be

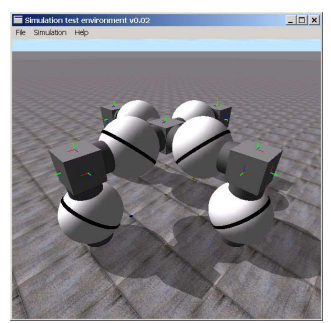

(a)

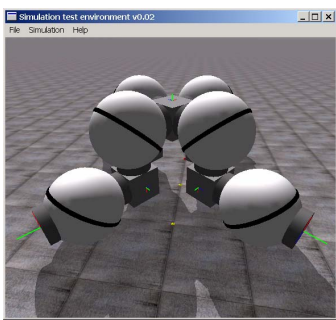

(b)

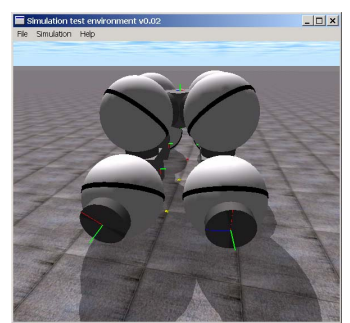

(c)

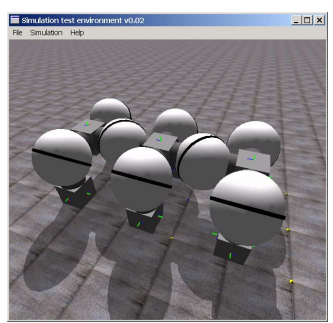

(d)

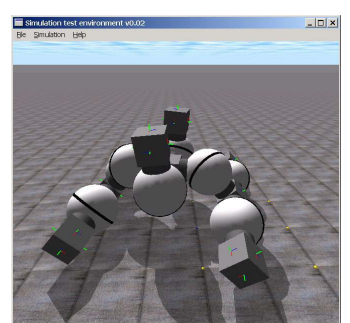

(e)

Figure 7. Kinematical reconfiguration from Quadruped to Rover (a,b,c) and from Hexapod to Rover $(\mathrm{d}, \mathrm{e})$ 


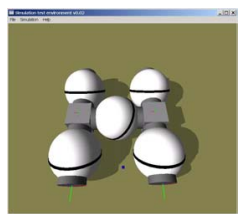

(a)

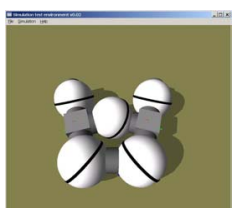

(b)

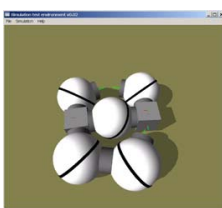

(c)

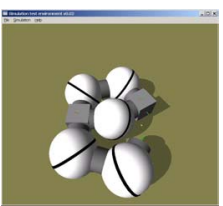

(d)

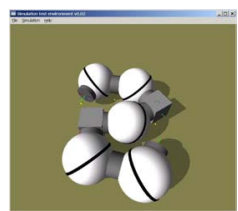

(e)

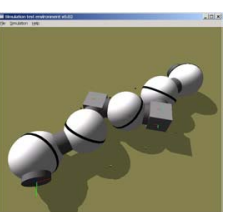

(f)

Figure 8. Stages of reconfiguration from Rover to Snake. (a) rover configuration, (b) connecting both south wheels, (c) disconnecting the southeast wheel from the east cube, (d) connecting both north wheels, (e) disconnecting the northwest wheel from the west cube, (f) expanding in snake configuration.

passive: active connector can connect to active and passive ones, but passive connector can only connect to active ones. This permits to reduce the number of latch mechanisms on the cubes and to gain place for other devices. Indeed, the Polybot connection mechanism without latch provides an efficient passive connector. All the shown configurations and reconfigurations can be done using 5 passive connectors for the cubes. However this limits the reconfiguration ability for more complex cases, since two cubes can not be connected together. We will determine in further analysis the optimal connectivity of the cube for a set of representative use-cases.

Serially connected balls (see Figure 8 b) have a redundant revolute mobility at their connecting plates. We can eliminate this redundancy by using an universal joint motion for the balls. This reduces the actuators number to two per ball. A compact mechanism described in [15] permits to implement an efficient universal joint within a ball shape (see Fig. 5 and 9 in [15]). The described mechanism can perform in-plane bending (in a range of 180 degree) and orienting motion. When the joint is straight an orienting motion can be used to produce a rolling motion of the ball. Almost all the shown configuration and reconfiguration can be done using 2 DOF balls; only the transition from Fig. $7 \mathrm{~b}$ to Fig. 7c is not possible. Moreover, a universal joint prevents twisting between the two plates of a ball, thus it permits to pass signal and power wires. Further mechanical analysis will be necessary to choose the number of actuators ( 2 or 3 ), as well as the exact size of the modules.

\section{CONCLUSION}

We have presented an original design for SRS modules which can be used as wheels and as ball or universal joints. This allows making multi-modes locomotion robots for exploration and transport, as well as manipulators. Further studies will be necessary to determine the actuation design of the balls and the best connectivity of the cubes.

\section{REFERENCES}

[1] Keith Kotay and Daniela Rus, "Locomotion versatility through selfreconfiguration", Robotics and Autonomous Systems. 26 (1999), 217232.

[2] Mark Yim, "Locomotion with a unit-modular reconfigurable robot", PhD Thesis, Standford University 1994.

[3] Steven Dubowsky, Phase I study of "Self-transforming robotic planetary explorers", Final Report, Field and Space Laboratory Department of Mechanical Engineering Massachusetts Institute of Technology 1999.

[4] S. Murata, et al., "M-TRAN: self-reconfigurable modular robotic system”, IEEE/ASME Trans. Mech. Vol. 7, No. 4, pp. 431-441, 2002.

[5] Cem Ünsal and Pradeep K. Khosla, "Solutions for 3-D selfreconfiguration in a modular robotic system: implementation and motion planning", Sensor Fusion and Decentralized Control in Robotic Systems III, 2000.
[6] John W. Suh, Samuel B. Homans and Mark Yim "Telecubes: mechanical design of a module for self-reconfigurable robotics", 2002 IEEE Int. Conf. on Robotics and Automation (ICRA).

[7] E.Yoshida, S.Murata, S.Kokaji, K.Tomita, H. Kurokawa, "Micro selfreconfigurable modular robot using shape memory alloy", Journal of Robotics and Mechatronics, Vol.13, No.2 212-219, 2001.

[8] Woo Ho Lee, Arthur C. Sanderson, "Dynamic rolling of modular robots" Proc. IEEE Int. Conf. on Robotics and Automation, April, San Francisco, pp. 2840-2846, 2000.

[9] Francesco Mondada1 et al., "SWARM-BOT: a swarm of autonomous mobile robots with self-Assembling capabilities" Technical report, LSA2 - I2S - STI, Swiss Federal Institute of Technology, Lausanne, Switzerland, 2002.

[10] Mark Yim, David Duff and Ying Zhang, "closed-chain motion with large mechanical advantage", Proc. IEEE/RSJ International Conference on Intelligent Robots and Systems 2001.

[11] Mark Yim. Kimon Roufas, David Duff, Ying Zhang, Sam Homans, "Modular reconfigurable robots in space applications", Autonomous Robot Journal, special issue for robots in space, Springer Verlag, 2003.

[12] David G. Duff, Mark Yim and Kimon Roufas "Evolution of PolyBot: a modular reconfigurable robot", Proc. of the Harmonic Drive Int. Symposium, Nagano, Japan, Nov. 2001, and Proc. of COE/SuperMechano-Systems Workshop, Tokyo, Japan, Nov. 2001.

[13] Mark Yim Ying Zhang Kimon Roufas David Duff, "Connecting and disconnecting for chain self-reconfiguration with PolyBot", IEEE/ASME Transactions on mechatronics, special issue on Information Technology in Mechatronics, 2003.

[14] Peter Will, Andrès Castaño, Wei-Min Shen, "Robot modularity for selfreconfiguration", Proc. SPIE Sensor Fusion and Decentralized Control II, pp. 236-245, Boston, Sep. 1999.

[15] Elie Shammas, Alon Wolf, H. Ben Brown Jr., Howie Choset, "New joint design for three-dimensional hyper redundant robots", Proc. IEEE/RSJ Int. Conference on Intelligent Robots and Systems, October 2003.

[16] Michel Dubois, Yann Le Guyadec, Dominique Duhaut, "Control of interconnected homogeneous atoms: language and simulator", Proc. CLAWAR International Conference On Climbing and Walking Robots, September 2003. 